\title{
Concurrent engineering approach in the development of composite products: a review
}

\begin{abstract}
Concurrent Engineering (CE) is regarded as a systematic design approach which integrates concurrent design of product with the related processes which is able to accomplish product that can be produced at lower cost, shorter time and with higher quality and this achievement was termed as cost, time and quality (CTQ) improvement. Since its establishment, CE philosophy was well implemented in product development with traditional materials such as metals but up to date, the work on CE in composite product development is still limited. Hence, a review on the implementation of Concurrent Engineering (CE) approach in the development of composite products is presented in this paper which includes review of various studies of $\mathrm{CE}$ techniques in composite product development. In addition, the relationship between $\mathrm{CE}$ and Pugh total design method is discussed in the context of composite design. Moreover, publications related to materials selection, life cycle analysis and sustainability issues of composite materials are also reviewed whereby a section is devoted to highlight previous work on materials selection using Analytical Hierarchy Process method. It was observed that materials selection of composite materials is a very important activity as far as CE in composite product development. The use of various techniques and computer aided materials selection tools such as Analytical Hierarchy Process has helped designers to select the most optimum composite materials for engineering components. Furthermore, based on current trends in composites product development, the role of CE is expected to be more crucial to assist composites designers in achieving the design requirements from various stakeholders effectively and efficiently considering the expanding range of composite materials availability as well as realizing new potential for biocomposites applications through introduction of innovative alternative problem solving methods as part of the CE family.
\end{abstract}

Keyword: Composites; Design for manufacturability; Concurrent engineering; Design for sustainability; Simultaneous engineering 